\title{
Identification de paramètres et contrôle vectoriel en temps réel d'un moteur asynchrone dans l'environnement Matlab/Simulink/dSPACE
}

\author{
C. Versèle, O. Deblecker et J. Lobry \\ Service de Génie Electrique - Faculté Polytechnique de Mons, Bd Dolez, 31 B-7000 Mons \\ christophe.versele@fpms.ac.be, olivier.deblecker@fpms.ac.be, jacques.lobry@fpms.ac.be
}

\begin{abstract}
RESUME - Cet article présente un projet destiné aux étudiants de premier Master ingénieur à finalité «Energie Electrique ». Durant ce projet, ces derniers doivent déterminer les paramètres et implanter une structure de contrôle vectoriel en temps réel d'une machine asynchrone alimentée par un onduleur de tension à IGBT dans l'environnement Matlab/Simulink/dSPACE. Grâce à la plate-forme expérimentale instrumentée à l'aide du matériel dSPACE, les étudiants peuvent se focaliser sur le schéma de commande de l'application sans perdre trop de temps sur des détails concernant la programmation des microprocesseurs constituant la carte de contrôle utilisée.
\end{abstract}

MOTS CLES - Machine asynchrone, Détermination de paramètres, Commande vectorielle, Environnement Matlab/Simulink/dSPACE.

\section{INTRODUCTION}

La robustesse, le faible coût, les performances ainsi que la simplicité de conception, de construction et d'entretien de la machine asynchrone (MAs) font qu'elle a la faveur des industriels, pour de nombreuses applications, depuis son invention par Tesla à la fin du dix-neuvième siècle.

Malheureusement, ces nombreux avantages s'accompagnent d'une grande complexité physique liée aux interactions entre le stator et le rotor. Dès lors, sa commande dynamique exige des algorithmes de contrôle complexe à l'opposé de sa simplicité structurale.

Durant ces dernières décennies, de nombreuses techniques de contrôle de la machine asynchrone, telles que les techniques de contrôle à flux orienté ou de contrôle vectoriel, ont vu le jour et nous permettent actuellement de jouir des avantages de la machine asynchrone pour des applications où la vitesse variable est indispensable.

Le principe de la commande vectorielle consiste à rendre le contrôle de la machine asynchrone analogue à celui de la machine à courant continu à excitation indépendante où il existe un découplage naturel entre le contrôle du flux (contrôlé par le courant d'excitation) et le contrôle du couple (contrôlé par le courant d'armature).

Dans cet article, nous présentons un projet ${ }^{1}$ dans lequel les étudiants doivent implémenter une structure de contrôle vectoriel dans l'environnement temps réel Matlab/Simulink/dSPACE.

Pour ce faire, ils doivent tout d'abord déterminer, de manière fine, les paramètres de la machine asynchrone utilisée sur la base d'une procédure de détermination fondée sur la structure de contrôle vectoriel. Ils doivent

\footnotetext{
${ }^{1}$ Ce projet a été récompensé par le prix GREPES 2007 (Groupe de Recherche en Electronique de Puissance pour Environnement Sévère) dont l'objectif est de récompenser un mémoire de fin d'études en électronique de puissance se distinguant par l'originalité de la méthode et des résultats.
}

ensuite calculer les gains des correcteurs proportionnel - intégral (PI) présents dans les différentes boucles de régulation constituant la structure de commande vectorielle. Enfin, ils réalisent différents essais en régulation et en poursuite de vitesse à vide et en charge.

\section{PLATE-FORME EXPERIMENTALE}

Le laboratoire de Génie Electrique de la Faculté Polytechnique de Mons s'est récemment doté de trois plates-formes expérimentales instrumentées dSPACE destinées à l'enseignement de la théorie générale des machines électriques, de l'électronique de puissance et de la commande des machines électriques. Chacune d'entre-elles est constituée de trois parties essentielles :

$>$ la partie «PUISSANCE »;

$>$ la partie «CONTRÔLE - COMMANDE »;

$>$ un environnement de mesures.

La figure 1 présente un schéma synoptique de la plateforme expérimentale utilisée dans le cadre de ce projet.

\section{A. Partie «PUISSANCE »}

La partie «PUISSANCE » consiste en un onduleur de tension à IGBT et deux machines: une machine asynchrone d'une puissance de $5,5 \mathrm{~kW}$ et une machine à courant continu à excitation indépendante d'une puissance de 14,6 kW. Celles-ci sont accouplées et la machine à courant continu est utilisée comme charge du moteur asynchrone.

\section{B. Partie « CONTRÔLE - COMMANDE »}

La partie «CONTRÔLE - COMMANDE » s'articule autour de la carte de contrôle DS1104 R\&D Controller Board développée par la société allemande dSPACE et logée dans un ordinateur. Cette carte de contrôle se compose de deux processeurs. Le processeur maître permet de gérer l'application tandis que le processeur esclave, un DSP («Digital Signal Processor ») de marque TEXAS INSTRUMENT (type TMS320F240), 


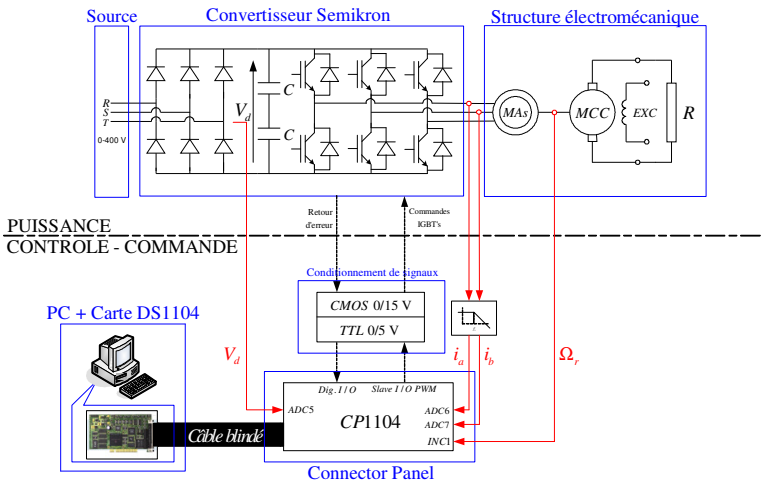

Figure 1 - Schéma synoptique de la plate-forme expérimentale

génère les signaux de commandes MLI (Modulation de Largeur d'Impulsion) en logique TTL 0/5 V. Ceci constitue la partie «hardware » de dSPACE.

La partie «software » se compose, quant à elle, de deux logiciels. Le premier, Matlab/Simulink, permet une programmation aisée de l'application temps réel sous Simulink par utilisation de blocs spécifiques (appartenant à la «toolbox Real Time Interface (RTI)») permettant de configurer les entrées/sorties de la carte DS1104. Le second logiciel, ControlDesk, permet de charger le code du programme sur la carte (écrit sous forme graphique dans Simulink, compilé et transformé en code C), de créer un environnement d'expérimentation complet et notamment une interface graphique de commande du procédé temps-réel, de traiter les données et de les enregistrer sous un format compatible avec Matlab (en vue d'un traitement ultérieur) ou encore de suivre en temps-réel l'évolution des données mesurées ou calculées à l'aide d'afficheurs graphiques ou digitaux.

\section{Environnement de mesures}

L'échange d'informations entre les deux parties décrites supra s'effectue par l'intermédiaire d'un boîtier externe de raccordement (Connector Panel CP1104 de la société dSPACE) relié à la carte via un câble blindé et recevant les signaux analogiques par l'intermédiaire de connecteurs BNC, d'une interface de conditionnement des signaux de commande MLI et des éventuels signaux d'erreurs renvoyés par le convertisseur Semikron et d'un environnement de mesure constitué de divers capteurs.

L'interface de conditionnement des signaux réalise la conversion de ces derniers de la logique TTL 0/5 V en logique CMOS $0 / 15 \mathrm{~V}$ et inversement. Cette modification est indispensable car la carte de contrôle DS1104 travaille avec des signaux en logique TTL $0 / 5 \mathrm{~V}$ tandis que ceux-ci doivent être en logique CMOS 0/15 V pour l'onduleur de tension.

L'environnement de mesure est constitué de capteurs LEM type LA55TP (capteurs de courant boucle fermée utilisant l'effet Hall) pour les mesures de courants, de capteurs LEM type LV100-500 (capteurs de tension de type boucle fermée utilisant l'effet Hall) pour les mesures de tensions et d'un codeur incrémental pour mesurer la vitesse de rotation du moteur. Enfin, les courants analogiques mesurés sont destinés à un traitement numérique et doivent donc être échantillonnés. Dès lors, afin d'éviter tout phénomène de repliement spectral, il est nécessaire d'insérer un filtre de garde (de fréquence de coupure estimée à 500 $\mathrm{Hz}$, un ordre de grandeur au-dessus de la fréquence fondamentale de $50 \mathrm{~Hz}$ ) entre chaque capteur et le convertisseur analogique/numérique.

\section{DEMARCHE PEDAGOGIQUE}

L'analyse du fonctionnement de la machine asynchrone alimentée via un onduleur de tension n'est pas simple à cause du comportement non linéaire de ces deux éléments [1]. De plus, le contrôle vectoriel d'un moteur asynchrone requiert des connaissances théoriques dans de nombreux domaines tels que les machines électriques, l'électronique de puissance, l'automatique, etc. Grâce à la plate-forme expérimentale instrumentée dSPACE, les étudiants peuvent se focaliser sur la détermination des paramètres et la structure de contrôle vectoriel du moteur asynchrone sans perdre trop de temps sur la programmation de l'application.

Le projet est divisé en plusieurs parties. Premièrement, un enseignement concernant la modélisation de Park de la machine asynchrone, l'objectif et le principe du contrôle vectoriel ainsi que ses expressions générales de commande et sa structure, est donné aux étudiants, ceux-ci connaissant déjà le principe de fonctionnement d'une telle machine ainsi que son modèle en régime établi (équations en substituts complexes). Dans un second temps, les étudiants sont amenés à programmer cette structure de contrôle, tout d'abord en simulation sous Simulink, ensuite dans l'environnement temps réel dSPACE, et à l'utiliser afin de déterminer les paramètres de la machine asynchrone. Pour ce faire, les références bibliographiques [2] et [3] leur sont fournies et décrivent étape par étape la procédure à suivre. Les paramètres étant maintenant connus, les étudiants peuvent aisément dimensionner les correcteurs PI présents dans la structure de contrôle et effectuer des essais expérimentaux en régulation et poursuite de vitesse. Enfin, un rapport écrit reprenant les étapes principales du projet leur est demandé.

Un volume horaire de 25 heures est consacré à la réalisation de ce projet proposé à des groupes constitués de 2 à 3 étudiants. Le volume horaire se répartit en 15 heures de travail expérimental et 10 heures de rédaction du rapport.

\section{A. Modèle de la machine asynchrone}

A partir du schéma équivalent de la machine asynchrone dans le repère de Park [4], présenté à la figure 2, nous obtenons les équations suivantes [5] : 
$v_{s d}=R_{s} i_{s d}+\frac{d \phi_{s d}}{d t}-\omega_{1} \phi_{s q}$

$v_{s q}=R_{s} i_{s q}+\frac{d \phi_{s q}}{d t}+\omega_{1} \phi_{s d}$

$v_{r d}=0=R_{r} i_{r d}+\frac{d \phi_{r d}}{d t}-\left(\omega_{1}-P \Omega_{r}\right) \phi_{r q}$

$v_{r q}=0=R_{r} i_{r q}+\frac{d \phi_{r q}}{d t}+\left(\omega_{1}-P \Omega_{r}\right) \phi_{r d}$

$\phi_{s d}=L_{s} i_{s d}+M i_{r d}$

$\phi_{s q}=L_{s q} i_{s q}+M i_{r q}$

$\phi_{r d}=L_{r} i_{r d}+M i_{s d}$

$\phi_{r q}=L_{r} i_{r q}+M i_{s q}$

où :

$v_{s d}$ et $v_{s q}$ (respectivement $v_{r d}$ et $v_{r q}$ ) sont les tensions statoriques (respectivement rotoriques) d'axes d et $\mathrm{q}$; $>i_{s d}$ et $i_{s q}$ (respectivement $i_{r d}$ et $i_{r q}$ ) sont les courants statoriques (respectivement rotoriques) d'axes $\mathrm{d}$ et $\mathrm{q}$; $>\phi_{s d}$ et $\phi_{s q}\left(\right.$ respectivement $\phi_{r d}$ et $\left.\phi_{r q}\right)$ sont les flux statoriques (respectivement rotoriques) d'axes d et q ; $>\omega_{1}$ est la vitesse angulaire des axes d et q dans le repère statorique et $\omega_{1}-P \Omega_{r}$ la vitesse des axes $\mathrm{d}$ et $\mathrm{q}$ dans le repère rotorique ;

$>\Omega_{r}$ est la vitesse (mécanique) de rotation de la machine asynchrone ;

$R_{s}$ (respectivement $R_{r}$ ) est la résistance statorique (respectivement rotorique);

$>L_{s}$ (respectivement $L_{r}$ ) est l'inductance statorique (respectivement rotorique) valant la somme de l'inductance de fuite statorique $l_{s}$ (respectivement rotorique $l_{r}$ ) et de l'inductance de magnétisation $M$.

Enfin, à ces équations, s'ajoutent encore l'expression du couple instantané :

$C_{e}=P \frac{M}{L_{r}}\left(\phi_{r d} i_{s q}-\phi_{r q} i_{s d}\right)$

et l'équation mécanique :

$J \frac{d \Omega_{r}}{d t}=C_{e}-C_{r}$

où :

$P$ est le nombre de paire(s) de pôles ;

$J$ est le coefficient d'inertie des masses tournantes ;

$C_{r}$ est la somme des couples résistants.

\section{B. Objectif et principe de la commande vectorielle}

L'objectif de la commande vectorielle est de rendre la machine asynchrone capable de répondre efficacement
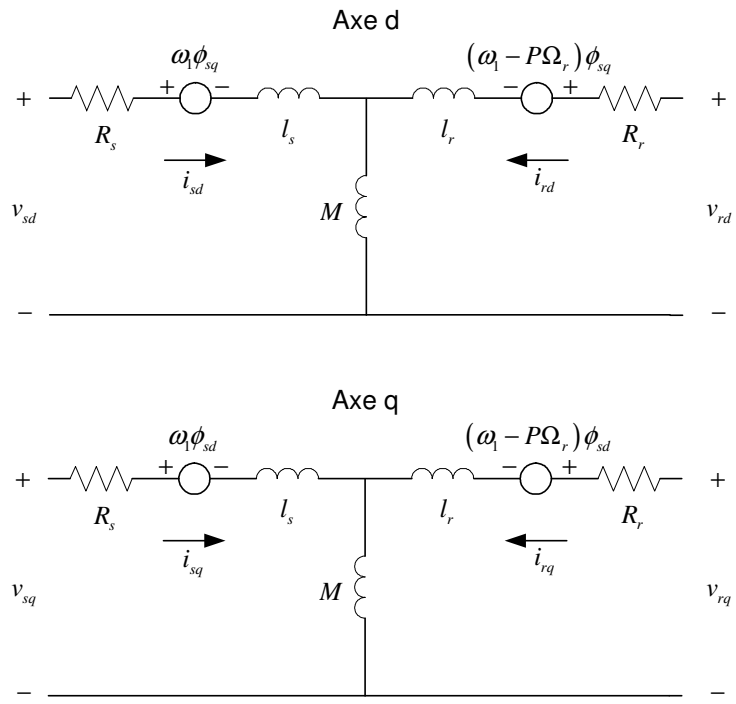

Figure 2 - Schéma équivalent de la machine asynchrone selon les axes $d$ et $q$ (repère de Park)

à des variations de consignes (position du rotor, couple, vitesse) dans une large gamme de points de fonctionnement et ce pour des applications nécessitant des performances dynamiques élevées.

Ce type de commande repose sur le contrôle instantané du couple et son principe consiste à rendre le fonctionnement de la machine asynchrone analogue à celui de la machine à courant continu à excitation indépendante où il existe un découplage naturel entre les contrôles du flux et du couple. En effet, dans une telle machine, le courant d'excitation permet de régler le flux inducteur dans l'axe polaire, tandis que le courant d'armature est utilisé pour contrôler le couple dans l'axe neutre (perpendiculaire à l'axe polaire).

Un choix adéquat du repère $(\mathrm{d}, \mathrm{q})$ de Park, choisi de façon à ce que l'axe $\mathrm{d}$ soit aligné avec la direction du vecteur flux rotorique, permet de réaliser un tel découplage. Nous parlons alors de contrôle vectoriel à flux rotorique orienté

Par ce choix de stratégie pour le contrôle vectoriel, la composante selon l'axe q du flux rotorique étant nulle, l'expression (9) du couple devient :

$C_{e}=P \frac{M^{2}}{L_{r}} i_{m r} i_{s q}$

où $i_{m r}$ est le courant magnétisant rotorique défini par :

$\phi_{r d}=M i_{m r}$

A partir des équations (3), (7) et (12), nous pouvons exprimer le courant magnétisant rotorique en fonction du courant statorique d'axe d. Nous obtenons alors :

$i_{m r}+T_{r} \frac{d i_{m r}}{d t}=i_{s d}$

où $T_{r}$ est la constante de temps rotorique (définie $\operatorname{par} T_{r}=L_{r} / R_{r}$ ).

Nous remarquons donc, grâce aux équations (11) et (13), que la composante directe du courant statorique 
$i_{s d}$ contrôle le flux tandis que la composante en quadrature de ce courant $i_{s q}$ contrôle le couple. Le découplage artificiel (car purement mathématique et non physique) recherché entre le flux et le couple est ainsi réalisé vu que les courants $i_{s d}$ et $i_{s q}$ sont en quadrature l'un par rapport à l'autre.

Remarquons encore que le contrôle vectoriel à flux rotorique orienté requiert, à chaque instant, la connaissance du flux rotorique (en module et en phase) et que celui-ci peut être obtenu par mesure ou plus souvent par estimation. C'est cette seconde solution qui est retenue dans le cadre du projet et il est demandé aux étudiants de construire cet estimateur. A partir de l'équation (13), on montre aisément que le module du courant magnétisant rotorique (qui est une image du flux rotorique) s'obtient en filtrant le courant statorique d'axe d par un filtre passe-bas de constante de temps $T_{r}$. De plus, on montre, par les équations (4) et (8), que la vitesse angulaire $\omega_{1}$ du repère tournant de Park vaut :

$\omega_{1}=P \Omega_{r}+\frac{i_{s q}}{T_{r} i_{m r}}$

Pour obtenir la phase $\mu$ du courant magnétisant rotorique (correspondant à la position de l'axe $\mathrm{d} d u$ repère de Park), il suffit donc d'intégrer cette relation. La figure 3 présente l'estimateur implanté dans l'environnement Matlab/Simulink/dSPACE.

\section{Expressions générales de la commande}

Dans le cadre de ce projet, nous pilotons l'onduleur en tension. Dès lors, les grandeurs réglantes pour le flux rotorique et le couple sont les tensions statoriques d'axes d et q ( $v_{s d}$ et $\left.v_{s q}\right)$. Celles-ci s'expriment par [5] : $v_{s d}=R_{s}\left[i_{s d}+T_{s}\left(\sigma \frac{d i_{s d}}{d t}+(1-\sigma) \frac{d i_{m r}}{d t}-\sigma \omega_{1} i_{s q}\right)\right]$

et

$$
v_{s q}=R_{s}\left[i_{s q}+T_{s}\left(\sigma \frac{d i_{s q}}{d t}+(1-\sigma) \omega_{1} i_{m r}+\sigma \omega_{1} i_{s d}\right)\right]
$$

où $T_{s}$ (définie par $T_{s}=L_{s} / R_{s}$ ) et le coefficient $\sigma$ (défini par $\sigma=1-M^{2} / L_{s} L_{r}$ ) sont respectivement la constante de temps statorique et le coefficient de dispersion de Blondel. Ce dernier donne une estimation globale des inductances de fuite dans la machine.

En examinant les expressions (15) et (16), nous constatons la présence de termes de couplage entre les axes d et q. Afin de limiter l'effet d'une entrée sur une seule sortie, et donc d'éliminer le couplage entre les axes $\mathrm{d}$ et $\mathrm{q}$, nous insérons un circuit de découplage à la sortie des régulateurs PI (voir figure 4). Le principe régissant ce circuit consiste à définir de nouvelles variables de commande $v_{s d}^{\prime}$ et $v_{s q}^{\prime}$.

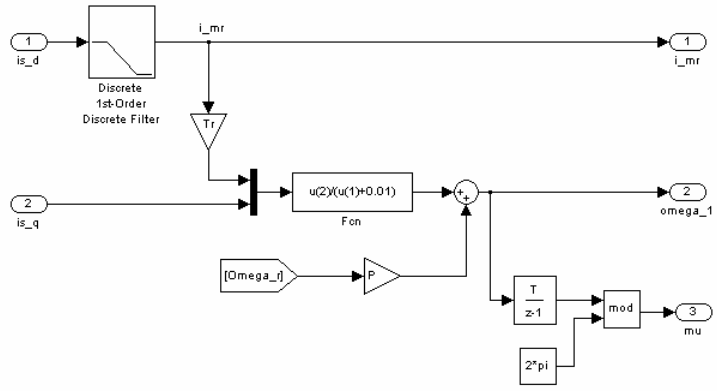

Figure 3 - Estimateur de flux rotorique

Celles-ci sont telles que $v_{s d}^{\prime}$ n'agit que sur le courant $i_{s d}$ et $v_{s q}^{\prime}$ sur le courant $i_{s q}$ et sont définies par :

$v_{s d}^{\prime}=R_{s}\left[i_{s d}+\sigma T_{s} \frac{d i_{s d}}{d t}\right]$

et

$v_{s q}^{\prime}=R_{s}\left[i_{s q}+\sigma T \frac{d i_{s q}}{d t}\right]$

\section{Structure de contrôle vectoriel}

La figure 4 présente la structure de contrôle vectoriel à flux rotorique orienté. Ce schéma correspond à une commande vectorielle de type direct dans laquelle le flux rotorique est asservi à une valeur de consigne. Celle-ci se compose de quatre régulateurs de type PI

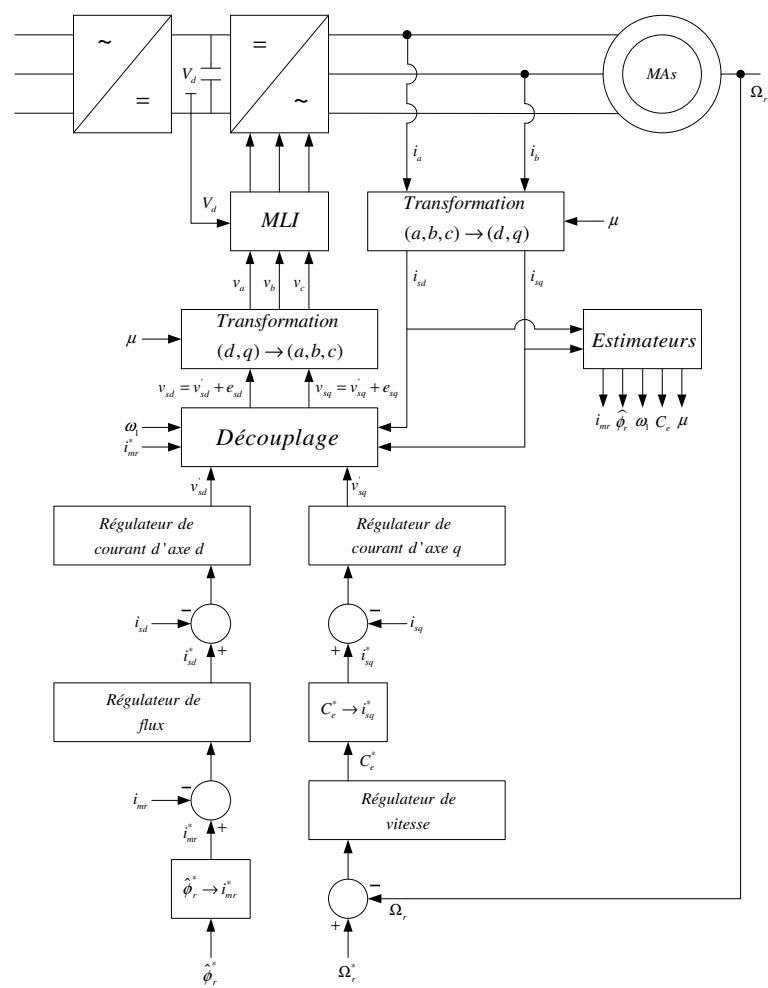

Figure 4 - Structure de contrôle vectoriel à flux rotorique orienté 
répartis en deux boucles de contrôle rendues indépendantes l'une de l'autre par découplage. La première boucle agit sur le flux et se compose du régulateur de flux ainsi que d'une boucle interne de courant dont le correcteur PI agit sur le courant statorique d'axe d $\left(i_{s d}\right)$. La seconde boucle agit quant à elle sur le couple et se compose du régulateur de vitesse et d'une boucle interne de courant dont le correcteur PI agit sur le courant statorique d'axe q $\left(i_{s q}\right)$.

\section{E. Détermination des paramètres}

La procédure de détermination des paramètres est amplement décrite dans les références bibliographiques [2] et [3], fournies aux étudiants, et est fondée sur la structure de contrôle vectoriel présentée supra. Cette procédure se compose de huit étapes. Tout d'abord, les paramètres de la plaque signalétique de la MAs doivent lui être fournis. Dans une seconde étape, la résistance statorique est mesurée par un essai en courant continu. Ensuite, la constante de temps transitoire statorique est mesurée. Grâce à ces deux premiers essais, les correcteurs PI des deux boucles internes de courant peuvent être dimensionnés. Dans la cinquième étape, la constante de temps rotorique est estimée et sa valeur est vérifiée (car il s'agit du paramètre le plus important pour obtenir une estimation correcte du flux rotorique) durant la sixième étape au cours de laquelle l'inductance de magnétisation est également mesurée. Grâce à la constante de temps rotorique, nous pouvons dimensionner le correcteur de flux durant la septième étape. Enfin, les paramètres mécaniques de l'ensemble MAs et génératrice à courant continu (voir infra) sont déterminés et le correcteur de vitesse dimensionné.

La procédure utilisée permet donc d'initialiser la structure de contrôle vectoriel en dimensionnant les deux boucles de contrôle présentées à la figure 4 à partir des valeurs des paramètres et présente l'avantage de pouvoir être automatisée par rapport à des méthodes classiques de détermination des paramètres. Dès lors, elle évalue les paramètres de la machine asynchrone lors de chaque démarrage de la plate-forme expérimentale et ajuste les valeurs des gains proportionnel et intégral de tous les correcteurs PI en fonction des paramètres actuels de la machine qui peuvent varier au cours du temps, par exemple, par suite du vieillissement de celle-ci.

Tout d'abord, les cinq paramètres du schéma équivalent dans les axes $\mathrm{d}$ et $\mathrm{q}$ de la machine asynchrone (voir figure 2) sont déterminés expérimentalement. Les résultats obtenus sont présentés dans le tableau 1 et comparés aux résultats obtenus par des essais classiques (essais au synchronisme et à rotor bloqué). Comme nous pouvons le constater, les résultats obtenus par les deux méthodes sont très proches.

De plus, la procédure utilisée permet également de déterminer les paramètres mécaniques (coefficient d'inertie $J$, constante de temps mécanique $T_{m}$ et coefficient de frottement visqueux $f_{v}$ ) de l'ensemble moteur asynchrone et génératrice à courant continu. Les résultats obtenus sont présentés dans le tableau 2.

\section{F. Dimensionnement des correcteurs PI}

A partir des paramètres déterminés au point précédent, les étudiants sont maintenant en mesure de calculer les gains proportionnel $\left(K_{P}\right)$ et intégral $\left(K_{I}\right)$ des quatre correcteurs PI présents dans la structure de contrôle vectoriel. Pour ce faire, la technique de compensation des pôles par des zéros leur est brièvement expliquée et est utilisée.

Les gains des correcteurs PI des deux boucles internes de courant (qui sont identiques) se déterminent à partir des équations (17) et (18) tandis que ceux du correcteur de vitesse et du correcteur de flux se déterminent respectivement à partir des équations (10) et (13). Les valeurs de ces gains sont présentées dans le tableau 3 et sont obtenues en imposant des temps de réponse à cinq pourcents de $2,5 \mathrm{~ms}$ pour les boucles internes de courant, de $25 \mathrm{~ms}$ pour la boucle de flux et de $250 \mathrm{~ms}$ pour la boucle de vitesse. Le temps de réponse le plus faible est choisi de façon à être compatible avec la dynamique de l'onduleur qui est commandé en MLI avec une fréquence de $9 \mathrm{kHz}$ (période de découpage de 0,111 ms choisie égale à la période d'échantillonnage).

\begin{tabular}{|c|c|c|}
\hline Paramètres & $\begin{array}{c}\text { Essais } \\
\text { classiques }\end{array}$ & $\begin{array}{c}\text { Procédure fondée } \\
\text { sur la structure de } \\
\text { contrôle vectoriel }\end{array}$ \\
\hline$R_{s}$ & $0,805 \Omega$ & $1,2625 \Omega$ \\
\hline$R_{r}$ & $0,5679 \Omega$ & $0,415 \Omega$ \\
\hline$l_{s}$ & $0,004 \mathrm{H}$ & $0,0039 \mathrm{H}$ \\
\hline$l_{r}$ & $0,004 \mathrm{H}$ & $0,0039 \mathrm{H}$ \\
\hline$M$ & $0,1601 \mathrm{H}$ & $0,1640 \mathrm{H}$ \\
\hline
\end{tabular}

Tableau 1 - Valeurs des paramètres de la machine asynchrone

\begin{tabular}{|c|c|}
\hline Paramètres & Valeurs \\
\hline$J$ & $0,0731 \mathrm{~kg} \cdot \mathrm{m}^{2}$ \\
\hline$T_{m}$ & $2,1 \mathrm{~s}$ \\
\hline$f_{v}$ & $0,0348 \mathrm{~kg} \cdot \mathrm{m}^{2} / \mathrm{s}$ \\
\hline
\end{tabular}

Tableau 2 - Valeurs des paramètres mécaniques de l'ensemble moteur asynchrone et génératrice à courant continu

\begin{tabular}{|c|c|c|}
\hline & $K_{P}$ & $K_{I}$ \\
\hline Boucles internes de courant & 9,4579 & 1630,68 \\
\hline Correcteur de flux & 48,6 & 120 \\
\hline Correcteur de vitesse & 0,887 & 0,4224 \\
\hline
\end{tabular}

Tableau 3 - Gains proportionnel $\left(K_{P}\right)$ et intégral $\left(K_{I}\right)$ des correcteurs $P I$ 


\section{G. Essais expérimentaux}

La structure de contrôle vectoriel étant maintenant initialisée, les étudiants peuvent, après de concluants essais en simulation sous Simulink, réaliser quelques essais expérimentaux en régulation et en poursuite de vitesse. Les figures 5 et 6 présentent respectivement la programmation de ladite structure sous dSPACE et l'interface graphique associée à l'application réalisée sous ControlDesk.

La figure 7 présente la réponse du système à un échelon de vitesse de $1200 \mathrm{tr} / \mathrm{min}$ à vide appliqué à l'instant $\mathrm{t}=0 \mathrm{~s}$. Nous pouvons y voir la valeur de consigne et la réponse du système et constatons que les quatre grandeurs régulées (courants statoriques d'axe d et q, flux et vitesse) suivent correctement leurs consignes. Nous présentons également sur cette figure la norme de la tension statorique $V_{s}$, la norme du courant statorique $I_{s}$ ainsi que le couple estimé $C_{e}$.

La figure 8 présente les résultats expérimentaux en poursuite (à vide) pour le profil de vitesses présenté sur le premier graphique. De nouveau, nous constatons que les grandeurs régulées suivent correctement leurs consignes et, en particulier, que la vitesse suit bien son profil et que le flux est correctement régulé.

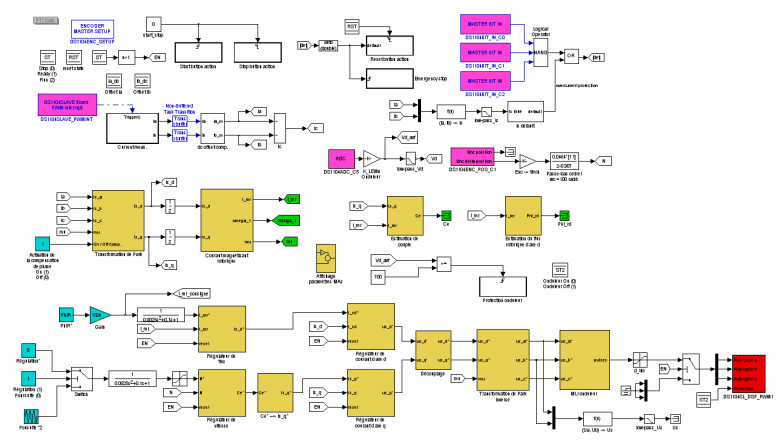

Figure 5 - Programmation de la structure de contrôle vectoriel sous dSPACE

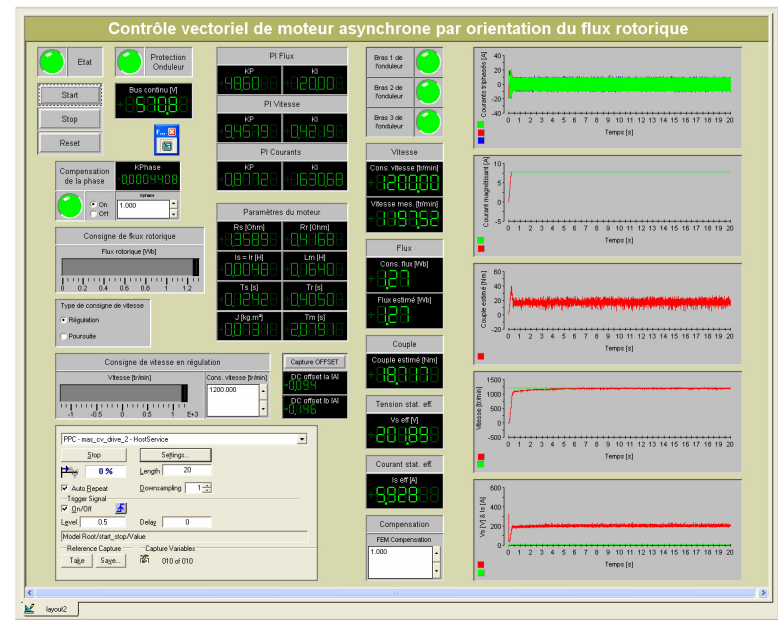

Figure 6 - Interface graphique réalisée sous ControlDesk
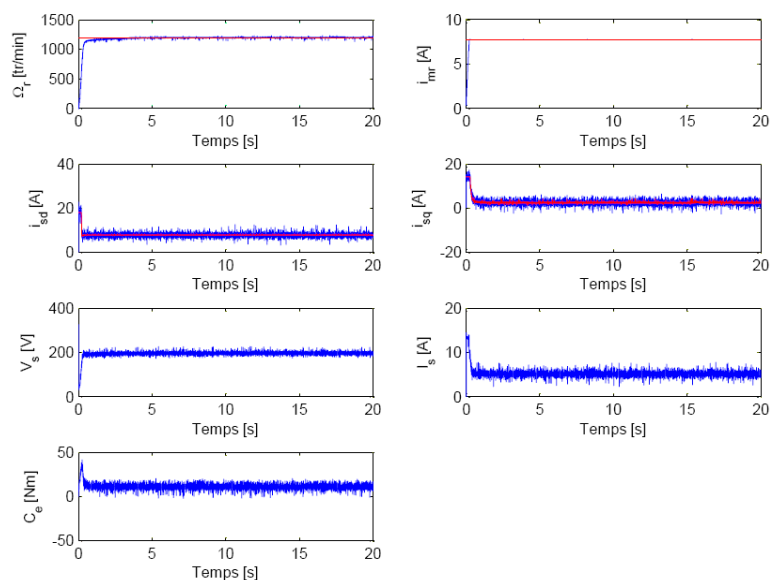

Figure 7 - Réponse à un échelon de vitesse à vide
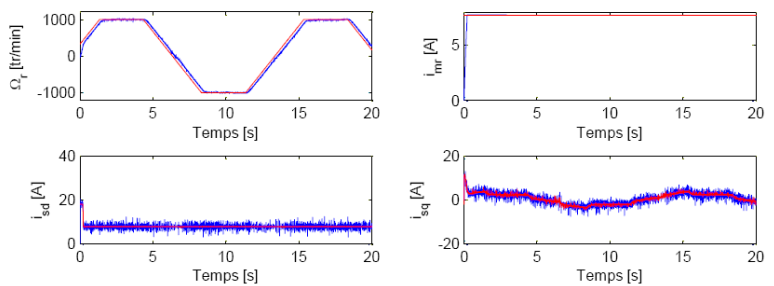

Figure 8 - Poursuite de vitesse à vide

\section{CONCLUSION}

L'environnement Matlab/Simulink/dSPACE offre un outil puissant et fort didactique pour l'enseignement de la détermination des paramètres et le contrôle des machines asynchrones. Grâce à cette plate-forme expérimentale, les étudiants peuvent aisément mettre en pratique un grand nombre de notions théoriques à travers un seul et unique projet. De plus, les résultats de ce projet ainsi que l'enthousiasme des étudiants poussent les auteurs à utiliser, de plus en plus, cet environnement pour d'autres projets ainsi que pour l'enseignement.

\section{BIBLIOGRAPHIE}

[1] Nahidmobarakeh B., Hénao H., Betin F. et Capolino G.A., «Une maquette modulaire pour l'initiation à la commande vectorielle des machines à induction », Actes du $4^{\text {ème }}$ Colloque sur l'Enseignement des Technologies et des Sciences de l'Information et des Systèmes (CETSISEEA'03), Toulouse, 13 et 14 novembre 2003, pp. 301-304.

[2] A. M. Khambadkone and J. Holtz, "Vector-Controlled Induction Motor Drive with a Self-Commissioning Scheme", IEEE Transaction on Industrial Electronics, Vol. 38, No. 5, pp. 322-327, octobre 1991.

[3] B. K. Bose, "Modern Power Electronics and AC Drives", Chapitre 8, Prentice Hall, New Jersey, 2001.

[4] Mohan N., « Advanced Electric Drives: Analysis, Control and Modeling using Simulink », MNPERE, 2001.

[5] Caron J.-P., Hautier J.-P., « Modélisation et commande de la machine asynchrone », Editions TECHNIP, 1995. 